\title{
ON THE COEFFICIENTS OF UNIVALENT LAURENT SERIES
}

\section{ZEEV NEHARI AND BINYAMIN SCHWARZ ${ }^{1}$}

While there exists a very extensive literature on the coefficients of Taylor series which are univalent in the unit circle, little attention seems to have been given to the corresponding problems for the coefficients of a Laurent series

$$
f(z)=\sum_{n=-\infty}^{\infty} a_{n} z^{n}, \quad 0<\rho<|z|<1,
$$

which is univalent for $\rho<|z|<1$. Most of the simple devices used in the treatment of univalent Taylor series cannot be profitably applied to the series (1), mainly because even the most elementary transformations of the series (1) affect its coefficients in a very complicated manner. However, there exists an elementary technique which can be applied to certain subclasses of (1) and can be made to yield sharp results. We shall illustrate its use in two cases: (a) for the subclass $R$ of univalent series (1) which have real coefficients $a_{n}$; (b) for the subclass $S$ of functions (1) which map $\rho<|z|<1$ onto a starlike domain $D$ with respect to the origin. By starlikeness is meant, as usual, that the intersection of $D$ and any rectilinear ray starting at the origin will be connected; obviously, the origin cannot belong to $D$, since otherwise $D$ would be simply-connected.

It is clear that if $f(z) \in R$, the sign of $\operatorname{Im}\{f(z)\}$ will be the same throughout each of the half-rings $\operatorname{Im}\{z\}>0, \rho<|z|<1$ and $\operatorname{Im}\{z\}$ $<0, \rho<|z|<1$, and that the sign will change if $z$ crosses the real axis. We shall normalize the functions of $R$ by the requirement that $\operatorname{Im}\{f(z)\}>0$ if $\operatorname{Im}\{z\}>0$; clearly, either $f(z)$ or $-f(z)$ will satisfy this normalization.

With this definition, we shall have $\sin \theta \operatorname{Im}\left\{f\left(r e^{i \theta}\right)\right\} \geqq 0(\rho<r<1$, $0 \leqq \theta \leqq 2 \pi)$ for any $f(z) \in R$. It follows that

$$
0 \leqq \frac{2}{\pi} \int_{0}^{2 \pi} \operatorname{Im}\left\{f\left(r e^{i \theta}\right)\right\} \sin \theta(1 \pm \cos \nu \theta) d \theta, \quad \nu=1,2, \cdots
$$

whence, by (1),

$0 \leqq 2\left(a_{1} r-a_{-1} r^{-1}\right) \pm\left[\left(a_{\nu+1} r^{\nu+1}-a_{-\nu-1} r^{-\nu-1}\right)-\left(a_{\nu-1} r^{\nu-1}-a_{-\nu+1} r^{\nu+1}\right)\right]$,

Received by the editors August 24, 1953.

${ }^{1}$ Work supported by the Office of Ordnance Research under Contract DA-23072-ORD-392. 
and therefore

$$
\left|a_{\nu+1} r^{\nu+1}-a_{-\nu-1} r^{-\nu-1}-\left(a_{\nu-1} r^{\nu-1}-a_{-\nu+1} r^{-\nu+1}\right)\right| \leqq 2\left(a_{1} r-a_{-1} r^{-1}\right) .
$$

Setting, without restricting generality, $a_{0}=0$, and using complete induction, we find that

$$
\left|a_{n} r^{n}-a_{-n} r^{-n}\right| \leqq n\left(a_{1} r-a_{-1} r^{-1}\right), \quad n=2,3, \cdots .
$$

This inequality holds for every $\rho<r<1$ and therefore, for reasons of continuity, also for $r=\rho, r=1$. It follows that

$$
\begin{aligned}
\left|a_{n}\right|\left(1-\rho^{2 n}\right) & =\left|a_{n}-a_{-n}+\rho^{n}\left(a_{-n} \rho^{-n}-a_{n} \rho^{n}\right)\right| \\
& \leqq\left|a_{n}-a_{-n}\right|+\rho^{n}\left|a_{n} \rho^{n}-a_{-n} \rho^{-n}\right| \\
& \leqq n\left(a_{1}-a_{-1}\right)+n \rho^{n}\left(a_{1} \rho-a_{-1} \rho^{-1}\right) .
\end{aligned}
$$

$a_{n}$ is thus subject to the inequality

$$
\left|a_{n}\right| \leqq \frac{n}{1-\rho^{2 n}}\left[a_{1}\left(1+\rho^{n+1}\right)-a_{-1}\left(1+\rho^{n-1}\right)\right], \quad n=2,3, \cdots .
$$

It will be observed that in the case of a Taylor series in the unit circle, that is, for $\rho=0, a_{-1}=0$, (4) reduces to the well known inequality $\left|a_{n}\right| \leqq n a_{1}$.

To find the corresponding inequality for the coefficients of negative powers, we write

$$
\begin{aligned}
\left|a_{-n}\left(\rho^{n}-\rho^{-n}\right)\right| & =\left|\rho^{n}\left(a_{-n}-a_{n}\right)+\left(\rho^{n} a_{n}-\rho^{-n} a_{-n}\right)\right| \\
& \leqq \rho^{n}\left|a_{-n}-a_{n}\right|+\left|\rho^{n} a_{n}-\rho^{-n} a_{-n}\right|
\end{aligned}
$$

and again apply (3) for $r=1$ and $r=\rho$. This leads to

$$
\begin{aligned}
\left|a_{-n}\right| \leqq \frac{n \rho^{n}}{1-\rho^{2 n}}\left[\rho a_{1}\left(1+\rho^{n-1}\right)-\rho^{-1} a_{-1}\left(1+\rho^{n+1}\right)\right], & \\
n & =2,3, \cdots .
\end{aligned}
$$

We now turn to the question as to when we may have equality in (4) and (5). Obviously, this can be the case only if there is equality in (2) for $r=\rho$ and $r=1$, since both (4) and (5) are based on these two special cases of (2). Now the integrand in (2) is non-negative and equality in (2) will therefore require that both $\operatorname{Im}\left\{f\left(e^{i \theta}\right)\right\}$ and $\operatorname{Im}\left\{f\left(\rho e^{i \theta}\right)\right\}$ are zero almost everywhere. This means that $f(z)$ must map $\rho<|z|<1$ onto the entire plane which is provided with two slits, one finite and one infinite, along the real axis.

The map of this function shows that there must be either a double pole at one of the points $\pm 1, \pm \rho$, or else two simple poles at two con- 
jugate points of $|z|=1$ or $|z|=\rho$. If it has a double pole at $z=1$, the integrand of (2) will remain continuous for $r=1$ (and, of course, also for $r=\rho$ ) and the integrand may indeed be evaluated on the boundary. The manipulations following (2) show that the question whether or not this function will give equality in (4) and (5) depends on the signs of the coefficients. This question can therefore not be decided without actually constructing a function according to the above specifications. It is easy to see that the function in question, say $f_{0}(z)$, is essentially an elliptic function and, except for a trivial transformation, it must be of the form

$$
f_{0}(z)=\wp(\log z ; i \pi, \log \rho),
$$

where $\varphi$ denotes the Weierstrassian elliptic function and $i \pi, \log \rho$ are its half-periods. Except for an additive constant, $f_{0}(z)$ has the Laurent expansion

$$
f_{0}(z)=\sum_{n=1}^{\infty} \frac{n \rho^{2 n} z^{-n}}{1-\rho^{2 n}}+\sum_{n=1}^{\infty} \frac{n z^{n}}{1-\rho^{2 n}} .
$$

It is easily verified that for the coefficients of $f_{0}(z)$ we have equality in (4) and (5). It can also be immediately confirmed that in the case of functions $f(z)$ for which $\operatorname{Im}\{f(z)\}<0$ if $\operatorname{Im}\{z\}>0$, (4) and (5) remain correct if the right-hand sides of these inequalities are replaced by their negative values. Summing up our results, we have the following theorem.

THEOREM I. Let

$$
f(z)=\sum_{n=-\infty}^{\infty} a_{n} z^{n}, \quad 0<\rho<|z|<1,
$$

be regular and univalent in $\rho<|z|<1$ and let the coefficients $a_{n}$ be real. Then

$$
\begin{aligned}
\left|a_{n}\right| \leqq \frac{n}{1-\rho^{2 n}}\left|a_{1}\left(1+\rho^{n+1}\right)-a_{-1}\left(1+\rho^{n-1}\right)\right|, & \\
n & = \pm 2, \pm 3, \cdots .
\end{aligned}
$$

This inequality is sharp for all values of $n$ concerned, as shown by the function (6) which satisfies all hypotheses.

REMARK. In our proof, we only made use of the fact that $\operatorname{Im}\{f(z)\}$ does not change its sign except when $z$ crosses the real axis. As in a similar case for Taylor series [1], our result will therefore also hold for typically-real Laurent series, that is, series whose sums are real if, 
and only if, $z$ is real.

It may also be worth commenting on the fact that both $a_{1}$ and $a_{-1}$ appear in the bound (7) for $a_{n}$. This is due to the circumstance that the family of univalent functions in a ring is not compact if only $a_{1}$ is kept fixed (or $a_{-1}$, for that matter). As an example, consider $f(z)=z-N z^{-1}$ which is univalent in $\rho<|z|<1$ if $N \geqq 1$, and let $N \rightarrow+\infty$.

We add here a remark regarding the sharpness of (7). As shown above, this inequality is sharp in the sense that, if $\rho$ is given, there exists a function whose coefficients $a_{1}, a_{-1}, a_{n}$ will lead to equality in (7). However, if $\rho$ and $a_{-1} / a_{1}$ are given independently (there are certain limitations; for instance, it follows from (3) that $a_{-1} \leqq \rho^{2} a_{1}$ if $f(z) \in R)$, the bound (7) will in general no longer be the best possible.

We now turn to the class $S$, which we shall further restrict by the assumption that $a_{0}=0$. As in the case of Taylor series, this results in somewhat simpler formulas. If $f(z) \in S$, our assumptions imply that $\arg \left\{f\left(r e^{i \theta}\right)\right\}(\rho<r<1)$ varies monotonically if $\theta$ grows from 0 to $2 \pi$; it may be either increasing or decreasing. Now it is easy to see that under these circumstances there must exist an angle $\theta_{0}$ such that the variation of arg $\left\{f\left(r e^{i \theta}\right)\right\}$ between $\theta=\theta_{0}$ and $\theta=\theta_{0}+\pi$ is exactly $\pi$. Indeed, since $\mid \Delta$ arg $\left\{f\left(r e^{i \theta}\right)\right\} \mid$ between $\theta_{0}$ and $\theta_{0}+2 \pi$ is $2 \pi$, the corresponding variation on one of the intervals $\theta_{0} \leqq \theta \leqq \theta_{0}+\pi, \theta_{0}+\pi \leqq \theta$ $\leqq \theta_{0}+2 \pi$ must be $\leqq \pi$ and on the other it is $\geqq \pi$. A monotonic increase of $\theta_{0}$ by the amount $\pi$ will reverse the role of the two intervals. Since, for constant $r(\rho<r<1), f\left(r e^{i \theta}\right)$ is a continuous function of $\theta$, it follows therefore that there exists an angle $\theta_{0}$ with the above properties. Hence, we may find suitable real numbers $\alpha, \beta$ such that the function

$$
g(z)=e^{i \alpha} f\left(e^{i \beta} z\right)
$$

takes real values for $z=r$ and $z=-r$. Obviously, $g(z) \in S$ and $\arg \left\{g\left(r e^{i \theta}\right)\right\}$ will thus vary monotonically with $\theta$. The function $g(z)$ (or $-g(z)$, obtained by replacing $\alpha$ by $\alpha+\pi$ ) is therefore such that $\operatorname{Im}\left\{g\left(r e^{i \theta}\right)\right\}>0$ for $0<\theta<\pi$ and $\operatorname{Im}\left\{g\left(r e^{i \theta}\right)\right\}<0$ for $\pi<\theta<2 \pi$. It resembles, in this respect, the functions of $R$ discussed before and, indeed, we may use a technique similar to that employed in the case of the class $R$.

If

$$
f(z)=\sum_{n=-\infty}^{\infty} a_{n} z^{n}, \quad g(z)=\sum_{n=-\infty}^{\infty} b_{n} z^{n}, \quad a_{0}=b_{0}=0,
$$

it follows from the definition of $g(z)$ that 


$$
\left|a_{n}\right|=\left|b_{n}\right| \text {. }
$$

From the properties of $g(z)$ just mentioned, we conclude that $\sin \theta \operatorname{Im}\left\{g\left(r e^{i \theta}\right)\right\} \geqq 0$, for $0 \leqq \theta<2 \pi$, whence by (8),

$$
\sin \theta \operatorname{Im}\left\{\sum_{n=1}^{\infty}\left(b_{n} r^{n}-b_{-n}^{*} r^{-n}\right) e^{i n \theta}\right\} \geqq 0 .{ }^{2}
$$

With the notation

$$
b_{n} r^{n}-b_{-n}^{*} r^{-n}=C_{n}=A_{n}+i B_{n}, \quad n \geqq 1, A_{n}, B_{n} \text { real, }
$$

this is equivalent to

$$
\begin{array}{r}
A_{1}+\sum_{n=1}^{\infty} \cos n \theta\left(A_{n+1}-A_{n-1}\right)+\sin n \theta\left(B_{n-1}-B_{n+1}\right) \geqq 0,0 \leqq \theta<2 \pi, \\
\left(A_{0}=B_{0}=0\right) .
\end{array}
$$

By a well known classical inequality for positive trigonometric series, it now follows that

$$
\left|A_{n+1}-A_{n-1}-i\left(B_{n-1}-B_{n+1}\right)\right| \leqq 2 A_{1}, \quad n=2,3, \cdots .
$$

Hence, by (10), $\left|C_{n+1}-C_{n-1}\right| \leqq 2 A_{1} \leqq 2\left|C_{1}\right|$. Using induction and the fact that $C_{0}$ may be taken to be zero, we find that $\left|C_{n}\right| \leqq n\left|C_{1}\right|$ for $n=2,3, \cdots$. In view of $(10)$, this yields

$$
\left|b_{n} r^{n}-b_{-n}^{*} r^{-n}\right| \leqq n\left|b_{1} r-b_{-1}^{*} r^{-1}\right|
$$

and thus, by (9),

$$
|| a_{n}\left|r^{n}-\right| a_{-n}\left|r^{-n}\right| \leqq n\left(\left|a_{1}\right| r+\left|a_{-1}\right| r^{-1}\right), \quad n=2,3, \cdots .
$$

This inequality is satisfied for every $\rho<r<1$ and thus also for $r=\rho$, $r=1$. Hence,

$$
\begin{aligned}
\left(1-\rho^{2 n}\right)\left|a_{n}\right| & \leqq|| a_{n}|-| a_{-n}||+\rho^{n}|| a_{-n}\left|\rho^{-n}-\right| a_{n}\left|\rho^{n}\right| \\
& \leqq n\left[\left|a_{1}\right|\left(1+\rho^{n+1}\right)+\left|a_{-1}\right|\left(1+\rho^{n-1}\right)\right],
\end{aligned}
$$

and we have the following result.

THEOREM II. If

$$
f(z)=\sum_{n=-\infty}^{\infty} a_{n} z^{n}, \quad \rho<|z|<1, a_{0}=0,
$$

maps $\rho<|z|<1$ onto a domain starlike with respect to the origin, then

2 Asterisks denote complex conjugates. 


$$
\begin{aligned}
\left|a_{n}\right| \leqq \frac{n}{1-\rho^{2 n}}\left[\left|a_{1}\right|\left(1+\rho^{n+1}\right)+\left|a_{-1}\right|\left(1+\rho^{n-1}\right)\right], & \\
n & = \pm 2, \pm 3, \cdots .
\end{aligned}
$$

That this inequality also holds for negative values of $n$ is seen by eliminating $\left|a_{n}\right|$ from the two inequalities obtained from (11) by setting $r=1$ and $r=\rho$, respectively.

If the Laurent series reduces to a Taylor series, (12) reduces to the well known sharp inequality $\left|a_{n}\right| \leqq n\left|a_{1}\right|$. In the general case, however, it is doubtful whether (12) is sharp. The function (6) belongs to $S$ and for geometric reasons it seems not unlikely that this function may also play the role of the extremal in our present problem. In this case, (12) would be superseded by the inequality (7), in which $a_{1}$ and $a_{-1}$ are replaced by $\left|a_{1}\right|$ and $\left|a_{-1}\right|$ respectively.

\section{REFERENCE}

1. W. Rogosinski, Über positive harmonische Entwicklungen und typisch-reelle Potenzreihen, Math. Zeit. vol. 35 (1932) pp. 93-121.

WASHINGTON UNIVERSITY 